\title{
Brain Tumor Segmentation using Multiobjective Fuzzy Clustering
}

\author{
Olfa Limam \\ Institut Supérieur d'Informatique, University Tunis EL Manar, Tunis, 2080, Tunisie \\ limemolfa@yahoo.fr
}

\begin{abstract}
The segmentation of magnetic resonance images plays a crucial role in medical image analysis because it extracts the required area from the image. Despite intensive research, it still remains a challenging problem and there is a need to develop an appropriate and efficient medical image segmentation method. In this paper, we propose a clustering approach for brain tumor segmentation to diagnose accurately the region of cancer. Applied to magnetic resonance image brain our method provides better identification of brain tumor.
\end{abstract}

Keywords: Brain tumor image segmentation, fuzzy clustering, multiobjective optimization, genetic algorithm.

\section{Introduction}

Image segmentation is an essential process in most medical image analysis, particularly in the study of some brain disorders. Interpretation process of complex medical structures, like tumor, cannot be identified without automatic image segmentation [1]. For example, the tumor portion of an image is defined as the irregular development of cells' growth inside the brain. Hence, image segmentation is needed to extract the required area from images [2]. As a common data analysis tool, clustering is a vital technique to segment a given data set into a distinct clusters such that similar patterns are assigned to a group, which is considered as a cluster [3]. Clustering works efficiently in discriminating between different tissues from medical images [4]. Conventional clustering methods assign points exclusively to one cluster. However, real medical image data sets present overlapping gray-scale intensities for different tissues [4]. In particular, borders between tissues are not clearly defined and memberships in the boundary regions are fuzzy. Fuzzy sets define the idea of uncertainty of belonging by a membership function [4]. Fuzzy clustering is considered to be the most suitable to model this situation in decision oriented applications such as tumor detection and tissue classification [5]. Fuzzy clustering technique provides a means of classifying pixel values with a good accuracy.

Several studies of brain tumor segmentation have been proposed in the literature. Methods based on statistical models such as Markov random models and Gaussian intensity models work well in normal brain image segmentation but not for abnormal tissues [6].

In pathological cases, methods based on classification techniques are widely used in image segmentation and have shown their robustness [6]. Fuzzy c-means (FCM) is the most popular clustering for image 
segmentation [1]. Li et al. [7] segmented brain images using FCM algorithm. Maksoud et al. [8] used Kmeans clustering technique integrated with FCM algorithm. It is followed by thresholding and level set segmentation stages to provide an accurate brain tumor detection. Pham and Prince [9] used also FCM to deal with magnetic resonance imaging (MRI). Udupa and Pnuam [10] used the fuzzy connectedness for abnormal tissue segmentation. Menon et al. [11] and Alsmadi [12] proposed a MRI brain image segmentation method using the hybridization of FCM and artificial bee colony algorithm.

Fuzzy clustering has been successfully applied, even though, it is greatly affected by noise and being very sensitive to its initialization [13].

MRI segmentation using thresholding is thought to be a very simple method to section an image that has dark backgrounds. Natarajan et al. [14] used thresholding based method for proficient recognition of a brain tumor image. Thapaliya et al. [15] used also thresholding for brain tumor image. Whereas, the determination of thresholding for the distribution of tissue intensities is very complex [1]. Therefore, segmentation techniques based on thresholding are simple and effective but they don't consider busyness of gray levels [16].

Region growing is also an important segmentation approach used for tumor detection. Weglinski et al. [17] proposed a region growing approach to segment brain tumor images. Won and Kim [18] proposed a similar technique to detect a brain ventricle in MRI brain images. Viji and JayaKumari [19] developed a texture based region growing technique for brain image segmentation. Gibbs et al. [20] used region growing for tumor volume determination. Lachmann et al. [21] used means of the texture analysis for brain tissue classification. Region growing is also effective but not a fully automatic segmentation of tumor tissues.

Numerous neural network based approaches have been proposed to segment brain images [22]. Hall et al. [23] used artificial neural network for segmentation of MRI brain images. Wells et al. [24] and Leemput et al. [25] used expectation-maximization (EM) for classification of MRI brain images. While, a common disadvantage of EM algorithm is the use of the normal distribution to model the intensity distribution which is untrue for noisy images [25].

Despite the potential of these approaches, some of them need manual guidance for their initialization with some manual learning [6]. In conclusion, fuzzy clustering is the most widely used technique [13].

However, fuzzy clustering methods optimizing a single objective fail to give satisfactory results since a single validity measure is not able to deal with different kinds of data sets. Moreover, the wrong selection of a validity measure leads to poor results [5]. Therefore, it is reasonable to consider more than one objective. Acharay et al. [26] used simulated annealing as the multiobjective optimization strategy for classification of tissue samples from cancer data sets. However, most multiobjective fuzzy clustering techniques are usually used in the segmentation of brain images but less for brain tumor detection [26]. Therefore, this paper proposes an adapted multiobjective fuzzy clustering algorithm for brain tumor segmentation of MRI images to accurately diagnose the region of cancer [27].

This paper is organized as follows. Section 2 details our proposed approach. Section 3 describes our experimental analysis and displays a visual comparison results with other fuzzy clustering techniques along with a quantitative comparison. Section 4 gives a conclusion and some perspectives. 


\section{The Multiobjective Fuzzy Clustering Model}

A brain tumor segmentation consists of separating the different tissues such as solid tumor, edema and necrosis from the normal brain tissues as same as the tissue classes of brain MRI, gray matter, white matter and cerebrospinal fluid [6].

We propose a multiobjective fuzzy clustering method which simultaneously optimizes the fuzzy spatial compactness of the clusters and the fuzzy spatial separation among the clusters. In brain MRI image, neighboring pixels have a strong correlation. Thus, we propose to incorporate the spatial information among objective functions to generate more accurate clusters. Moreover, we use FCM for encoding initial centers instead of randomly extracting them from the data set. For the final generation, our multiobjective approach produces a set of non-dominated solutions, from which the best solution is chosen to be the final image segmentation based on the I index measure. In the following section, we discuss the different steps of our proposed method.

\subsection{Feature extraction}

Original brain $\mathrm{MRI}$ is a gray-level image deficient to support fine features [2]. To obtain more useful features and improve the visual density, the proposed method adopts the standard RGB color space. In medical image segmentation, color features could be considered [28].

\subsection{Pattern proximity}

The pattern proximity is calculated using a distance function defined on pairs of patterns, namely the Euclidean distance [29]. In general, the distance between two pixels $x:\left(x_{1}, \ldots, x_{n}\right)$ and $y:\left(y_{1}, \ldots, y_{n}\right)$ in an Euclidean $n$-space is defined as follows:

$$
d(x, y)=|x-y|=\sqrt{\sum_{i=1}^{n}\left|x_{i}-y_{i}\right|^{2}}
$$

\subsection{Multiobjective fuzzy clustering algorithm}

NSGA-II is used as the underlying optimization multiobjective strategy. NSGA-II inputs are the data set, population size, maximum generation and an upper bound on the number of clusters. This algorithm requires a number of individuals as potential solutions to be initialized at the beginning of the algorithm.

\subsubsection{Population Initialization}

In NSGA-II based clustering, real-valued chromosomes representing the coordinates of the partitions (centers) are used. Centers encoded in a given chromosome in the initial population are computed using FCM instead of being randomly extracted from the data set to give a more robust partition. FCM produces $\mathrm{C}$ cluster centers and $\mathrm{C} \times \mathrm{N}$ membership matrix $\mathrm{U}(\mathrm{x})$. The FCM output is developed in a fuzzy representation where each pixel has a variable degree of membership to each of the output centers encoded in a chromosome. 


\subsubsection{Computation of the objectives}

NSGA-II fitness functions, fuzzy global spatial compactness and fuzzy spatial separation are simultaneously optimized and computed for each chromosome. To compute the objective functions, we extract the centers $V=\left\{v_{\mathbf{i}}\right\}$ for $i \in\{1, \ldots, C\}$ encoded in a given chromosome.

The first objective, global spatial compactness $\tau$, is given by :

$$
\tau=\sum_{i=1}^{C} \frac{\sigma_{i}}{n_{i}}=\sum_{i=1}^{C} \frac{\sum_{k=1}^{N} \boldsymbol{u}_{i k}^{{ }^{*} m} D\left(v_{i}, x_{k}\right)}{\sum_{k=1}^{N} \boldsymbol{u}_{i k}{ }^{* m}}
$$

where $\sigma_{i}$ denotes the variation, $\mathrm{D}\left(v_{i}, x_{k}\right)$ is the Euclidean distance between $\mathrm{i}^{\text {th }}$ cluster center and $\mathrm{k}^{\text {th }}$ data point, $m \in\{1, \infty\}$ is the fuzzy exponent and $n_{i}$ is the fuzzy cardinality of the ith cluster such that

$$
n_{i}=\sum_{k=1}^{N} \boldsymbol{u}_{i k}^{* m}, 1 \leq i \leq C
$$

The second objective, fuzzy spatial separation S, is defined as :

$$
S=\sum_{i=1}^{C} \sum_{i=1, j \neq i}^{C} u_{i j}^{* m} D\left(v_{i}, v_{j}\right) .
$$

The measure $\tau$ should be minimized while the fuzzy separation $S$ should be maximized, as follows

$$
\left\{\begin{array}{l}
\text { Min } \tau \\
\text { Max S }
\end{array}\right.
$$

We propose to integrate the relative locations of neighboring pixels assuming that a pixel has a dependent intensity to its surrounding pixels. However, a pixel is too small to represent a part of an image and it is affected by neighborhood intensity [1]. The notion of spatial coherence for brain tumors is carried out in order to segment different varieties of brain tumors. This is a challenging issue because tumors can appear in many different sizes and shapes [30]. The segmentation process is therefore decided not only by the pixel intensities but also by the neighboring pixels intensities. A pixel gets high membership value to a cluster when its neighborhood pixels have a higher membership value to that cluster [31].

The spatial function $h_{i k}$ represents the probability that pixel $x_{k}$ belongs to the $i^{\text {th }}$ clustering. The $N B\left(x_{k}\right)$ represents a square window centered where pixel $x_{k}$ is in the spatial domain. A window of $3 \times 3$ is used throughout this work. The modified membership function of the current pixel, that includes the spatial information, is defined as follows: 


$$
\boldsymbol{u}_{i k}^{*}=\frac{\boldsymbol{u}_{i k} \boldsymbol{h}_{i k}}{\sum_{j=1}^{C} \boldsymbol{u}_{j k} \boldsymbol{h}_{j k}},
$$

where $u_{i k}$ is computed as follows.

$$
u_{i k}=\frac{1}{\sum_{j=1}^{C}\left(\frac{D\left(v_{i}, x_{k}\right)}{D\left(v_{j}, x_{k}\right)}\right)^{\frac{2}{m-1}}}
$$

For $1 \leq \mathrm{i} \leq \mathrm{C} ; 1 \leq \mathrm{k} \leq \mathrm{N}$.

The spatial function is defined as:

$$
h_{i k}=\sum_{k \in N b\left(x_{k}\right)} g_{i k}
$$

where, for $\mathrm{I}=\{1, \ldots, \mathrm{C}\}$

$$
g_{i k}=\left\{\begin{array}{l}
1 \quad \text { if } u_{i k}=\max \left\{u_{l k}\right\}, \\
0 \quad \text { otherwise }
\end{array}\right\}
$$

\subsubsection{Genetic Operators}

Crowded binary tournament selection is adopted to generate the mating pool of chromosomes, followed by conventional crossover and mutation, is used here. The last part of the NSGA-II is its elitism operation, where the non dominated solutions among the parent and child populations are propagated to the next generation. This algorithm produces a large number of non-dominated solutions on the final Pareto optimal front.

\subsubsection{Optimal solution}

In the last stage of the proposed approach, it is obvious to choose the appropriate solution from the set of non-dominated solutions based on a validity measure namely the I index [32].

The pseudo code of our proposed algorithm is given in Algorithm 1.

\section{Algorithm 1}

1. Initialization process: FCM to compute the cluster centers of the partition encoded in chromosomes for the initial population.

2. Multiobjective optimization: Multiobjective clustering technique NSGA-II produces a set of near Pareto optimal solutions.

3. Partition validation: Maximum I index value. 


\section{Experimental Analysis}

In order to show the performance of our method, we conducted an experimental study on two simulated brain tumor image data sets representing two different tumor shapes. Moreover, we compare our segmented image to other images produced by competing algorithms respectively, k-means algorithm, histogram clustering and FCM clustering algorithm [2]. Then, a quantitative comparison is conducted between clustering algorithms based on performance accuracy and execution time.

The platform for conducting the experiments is a Core 2 Duo $2.10 \mathrm{GHz}$ laptop with $3 \mathrm{~GB}$ RAM using Windows 7 32bit. The program was implemented using MATLAB development environment. The simulated T-weighted MRI brain data sets were downloaded from Brainweb [33]. Brainweb provides a simulated brain database including a set of realistic MRI data volumes produced by an MRI simulator.

\subsection{Visual Comparison}

Figure 1 contains a low grade tumor, while, Figure 2 contains a large, partially enhancing tumor inside the brain stem. Figure 1 and Figure 2 (a) show the original MRI brain tumor image and (b) display the image segmented by our approach.

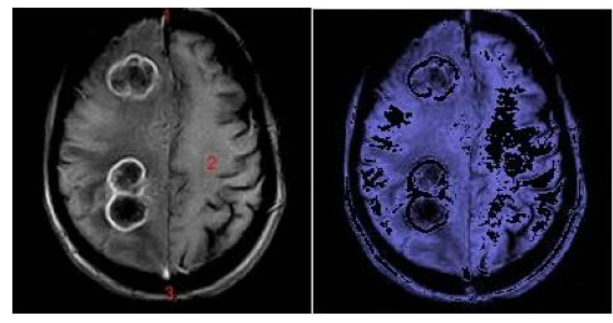

Figure 1 (a) Original MRI brain tumor image with a low grade tumour (b) Corresponding image segmented by our method
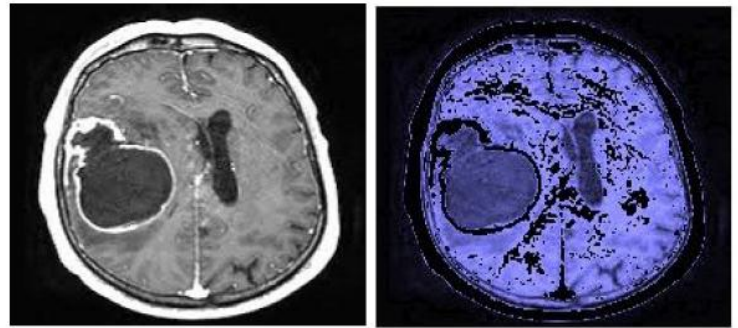

Figure 2 (a) Original MRI brain tumor image with a large grade tumour (b) Corresponding image segmented by our method

In Figures 1, 2, our method succeed to locate the tumor part of the brain and clearly identifying from the other part of the brain.

Figure 3 shows the segmentation results of four different clustering algorithms including our proposed method. Figure 3 shows (a) The original MRI brain image, (b) K-means segmented image, (c) Histogram segmented image, (d) FCM segmented image and (e) Our image segmented.

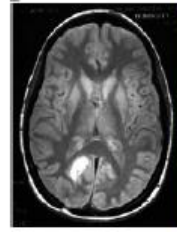

(a)

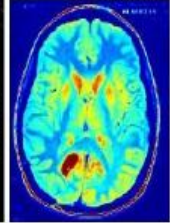

(b)

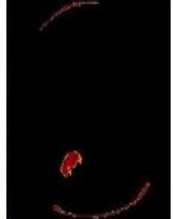

(c)

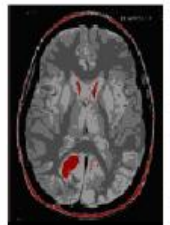

(d)

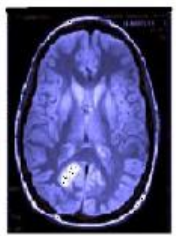

(e)

Figure 3 (a) The original MRI brain image, (b) k-means segmented image, (c) histogram segmented image, (d) FCM segmented image and (e) Our image segmented

From Figure 3, it can be noticed that the k-means and FCM clustering algorithms identify efficiently the different regions of the brain image, while the Histogram algorithm does not. Our proposed method succeed in identifying the different regions, showing the ability of fuzzy clustering for brain image segmentation and brain tumor detection. 
It can be seen through comparison that our method gives better result as compared to the original image.

In conclusion, based on experimental study, we show the effectiveness of our method in segmenting the brain tumor with different tumor grades. Also, our proposed method gives better results compared with three state-of-the-art algorithms: K-means, Histogram and FCM.

\subsection{Quantitative comparison for brain tumor images}

Moreover, to show the performance of our proposed method, we conducted a comparative study on two different brain tumor image data sets. Based on a performance measure namely accuracy [32], defined as follows

$$
\operatorname{Accuracy}(T, C)=\frac{a+b}{c} \times 100,
$$

The accuracy of segmentation is obtained as the ratio between the sum of correctly classified pixels to the total number of pixels [8], where $\mathrm{T}$ denotes the true clustering of data set based on domain knowledge, $\mathrm{C}$ the partition given by a clustering algorithm, a the number of pairs of points that belong to the same clusters in both $\mathrm{T}$ and $\mathrm{C}, \mathrm{b}$ the number of pairs of points that belong to different clusters in both $\mathrm{T}$ and $\mathrm{C}$, and $c$ the total number of pairs of points. A high value of accuracy ( $\geq 65 \%$ ) denotes better matching between $T$ and $C$ [35]. Thus, high values of accuracy mean that we have an optimal matching between the clustering solution and the known partition.

Also, our comparison is based on the execution time. Tables 1 and 2 show the results of a comparison between our proposed method with K-means (KM), Expectation Maximization (EM), Mean shift clustering technique (MS), FCM, K means integrated with fuzzy c means (KFCM), particle swarm optimization (PSO) [8] and our proposed method (PM), based on the performance accuracy and the execution time, respectively.

Table 1 The performance accuracy of fuzzy clustering algorithms for brain tumor images

\begin{tabular}{|l|l|l|l|l|l|l|l|}
\hline DS & KM & EM & MS & FCM & KFCM & PSO & PM \\
\hline (1. & 85.7 & 66.6 & 85.7 & 85.7 & 90.5 & 95.5 & 95.75 \\
\hline (3) & 95.06 & 95.06 & 95.06 & 100 & 100 & 100 & 100 \\
\hline
\end{tabular}

Table 1 illustrates the evaluation results using the performance measure of accuracy for different clustering algorithms. Where the accuracy refers to the percentage of correct predictions made by the model compared to the known partition in the test data.

The performance accuracy is enhanced compared to other algorithms. The performance accuracy results show the capacity of our proposed method in providing better results.

Table 2: The Execution time of fuzzy clustering algorithms for brain tumor images

\begin{tabular}{|l|l|l|l|l|l|l|l|}
\hline DS & KM & EM & MS & FCM & KFCM & PSO & PM \\
\hline (1) & 7.5 & 34.47 & 59.52 & 12.87 & 34.58 & 9.23 & 20.45 \\
\hline (3) & 4.34 & 32.06 & 6.89 & 3.46 & 6.26 & 6.01 & 3.56 \\
\hline
\end{tabular}


Table 2 shows the execution time (seconds). For the first brain image, KM takes less processing time but it does not give accurate results as in the second brain image. However, the obtained results indicate the success of our proposed method in detecting clearly the tumor site in the image. Therefore, our proposed method provides good results compared to other competing algorithms in terms of execution time.

\section{Conclusion}

This paper proposes an improved detection method of brain tumor using clustering technique. We used a multiobjective fuzzy clustering that integrates the spatial information and a robust initialization process. The proposed method is applied to segment MRI brain image for recognition of tumor. Both quantitative and visual comparison show the superiority of our proposed method which has the ability of finding tumor locations in MRI brain. Our method can be extended to detect tumors on other views of brain.

\section{REFERENCES}

[1] Shen Shan, Sandham William, Granat Malcolm, and Sterr Annette. Mri fuzzy segmentation of brain tissue using neighborhood attraction with neural-network optimization. IEEE Transactions on Information Technology in Biomedicine, 9(3):459-467, 2005.

[2] P.Tamije Selvy, V. Palanisamy, and T. Purusothaman. Performance analysis of clustering algorithms in brain tumor detection of mr images. European Journal of Scientific Research, 62(3):321-330, 2011.

[3] Suchita Yadav and Meshram Sachin. Brain tumor detection using clustering method. International Journal of Computational Engineering Research, 3(4):11-14, 2013.

[4] Miin-Shen Yang, Yu-Jen Hu, Karen Chia-Ren, and Charles Chia-Lee Lin. Segmentation techniques for tissue differentiation in mri of ophthalmology using fuzzy clustering algorithms. Magentic resonance Imaging, 20:173-179, 2002.

[5] Sanghamitra Bandyopadhyay, Anirban Mukhopadyay, and Ujjwal. Maulik. Combining multiobjective fuzzy clustering and probabilistic ann classifier for unsupervised pattern classification: Application to satellite image segmentation. IEEE on Evolutionary Computation, pages 877-883, 2008.

[6] Weibei Dou, Su Ruan, Daniel Bloyet, and Jean-Marc Constans. A framework of fuzzy information fusion for the segmentation of brain tumor tissues on mr images. Image and Vision Computing, 25(2):164-171, 2007.

[7] Chunlin Li, DB Goldgof, and LO Hall. Knowledge-based classification and tissue labeling of $\mathrm{mr}$ images of human brain. IEEE transactions on Medical Imaging, 12(4):740-750, 2002.

[8] Eman A. Abdel Maksoud, Mohammed Elmogy, and Rashid Mokhtar Al-Awadi. MRI brain tumor segmentation system based on hybrid clustering techniques. In Advanced Machine Learning Technologies and Applications - Second International Conference, AMLTA, pages 401-412, 2014.

[9] L. Dzung Pham and Jerry L. Prince. Knowledge-based classification and tissue labeling of $\mathrm{mr}$ images of human brain. IEEE transactions on Medical Imaging, 18(9):737-752, 1999. 
Olfa Limam; Brain Tumor Segmentation using Multiobjective Fuzzy Clustering. Transactions on Machine Learning and Artificial Intelligence, Volume 4 No 1 February (2016); pp: 58-67

[10] Jayram K. Udupa and K. Saha Punam. Fuzzy connectedness and image segmentation. Proceedings of the IEEE, 91:1649-1669, 2003.

[11] R Sivakumarl Neeraja R Menon, M Karnan. Brain tumor segmentation in mri image using unsupervised artificial bee colony and fcm clustering. International Journal of Computer Science and Management Research, 2:2450-2454, 2013.

[12] Mutasem K. Alsmadi. Mri brain segmentation using a hybrid artificial bee colony algorithmwith fuzzy-c mean algorithm. Journal of Applied Sciences, 15:100-109, 2015.

[13] Zhanshen Feng and Boping Zhang. Fuzzy clustering image segmentation based on particle swarm optimization. Telecommunication Computing Electronics and Control, 13(1):128-136, 2015.

[14] P. Natarajan, N. Krishnan, N.S. Kenkre, S. Nancy, and B.P. Singh. Tumor detection using threshold operation in mri brain images. IEEE International Conference on Computational Intelligence and Computing Research (ICCIC), pages 1-4, 2012.

[15] K. Thapaliya, , and Kwon Goo-Rak. Extraction of brain tumor based on morphological operations. 8th International Conference on Computing Technology and Information Management (ICCM), 20:515 - 520, 2012.

[16] Anam Mustaqeem, Ali Javed, and Tehseen Fatima. An efficient brain tumor detection algorithm using watershed thresholding based segmentation. Interntional Journal of Image, Graphics and Signal Processing, 10:34-39, 2012.

[17] Tomasz Weglinski and Anna Fabijanska. Brain tumor segmentation from mri data sets using region growing approach. Proceedings of VIIth International Conference on Perspective Technologies and Methods in MEMS Design, pages 185 - 188, 2011.

[18] Won Chulho and Kim Dong-Hun. Region growing method using edge sharpness for brain ventricle detection. SICE, Annual Conference, pages 1930-1933, 2007.

[19] K.S.A Viji and J. JayaKumari. Modified texture based region growing segmentation of mr brain images. IEEE Conference on Information Communication Technologies (ICT), pages 691-695, 2013.

[20] Peter Gibbs, David L. Buckley, Stephen J Blackband, and Anthony Horsman. Tumour volume determination from $\mathrm{mr}$ images by morphological segmentation. Physics in Medicine and Biology, 41:2437-2446, 1996.

[21] Frederic Lachmann and Christian Barillotr. Brain tissue classification from mri data by means of texture analysis. Medical Imaging VI: Image Processing, 72, 1992.

[22] D.M. Joshi, N. K. Rana, and V.M. Misra. Classification of brain cancer using artificial neural network. International Conference on Electronic Computer Technology (ICECT), pages 112-116.

[23] L.O Hall, A.M. Bensaid, L.P. Clarke, R.P. Velthuizen, and M.S. Silbige rand James C. Bezdek. A comparison of neural network and fuzzy clustering techniques in segmenting magnetic resonance images of the brain. IEEE Transactions on Neural Network, 3(5):672-682, 1992. 
[24] W. M. Wells, W. L. Grimson, R. Kikinis, and F. A . Jolesz. Adaptive segmentation of mri data. IEEE Transactions on Medical Imaging, 15(4):429-442, 1996.

[25] K. V. Leemput, F. Maes, D. Vandermeulen, and P. Suetens. Automated model-based tissue classification of $\mathrm{mr}$ images of the brain. IEEE Transactions on Medical Imaging, 18(10):897-908.

[26] Sudipta Acharya, Yamini Thadisina, and Sriparna Saha. Multi-objective clustering of tissue samples for cancer diagnosis. International Conference on Advances in Computing, Communications and Informatics, pages 1059-1064, 2014.

[27] Olfa Limam and Fouad Ben Abdelaziz. Multicriteria fuzzy clustering for brain image segmentation. 2013 5th International Conference on Modeling, Simulation and Applied Optimization (ICMSAO), pages 1019 1031, 2013.

[28] Bong Chin-Wei and Mandava Rajeswari. Multiobjective optimization approaches in image segmentation - the directions and challenges. International Journal of Advances in Soft Computing and Its Applications, 2(2):41-64, 2010.

[29] Anil Kumar Jain, M Narasimha Murty, and Patrick Joseph Flynn. Data clustering: A review. ACM Computing Surveys, 31(3):265-323, 1999.

[30] Marcel Prastawa, Elizabeth Bullitt, Sean Ho, and Guido Gerig. A brain tumor segmentation framework based on outlier detection. Medical Image Analysis, 8(3):275-283, 2004.

[31] Xiang-Yang Wang and Juan Bu. A fast and robust image segmentation using fcm with spatial information. Digital Signal Processing, 20:1173-1182, 2010.

[32] Jie Liu, Jigui Sun, and Shengsheng Wang. Pattern recognition: An overview. International Journal of Computer Science and Network Security IJCSNS, 6(6):57-60, 2006.

[33] Brainweb: Simulated brain database, 2011, available at http://www.bic.mni.mcgill.ca/brainweb.

[34] K.Y. Yeung and Walter L. Ruzzo. An empirical study of principal component analysis for clustering gene expression data. 2001.

[35] Maulik Ujjwal and Mukhopadhyay Anirban. A multiobjective approach to mr brain image. Applied Soft Computing $11,872-880$. 